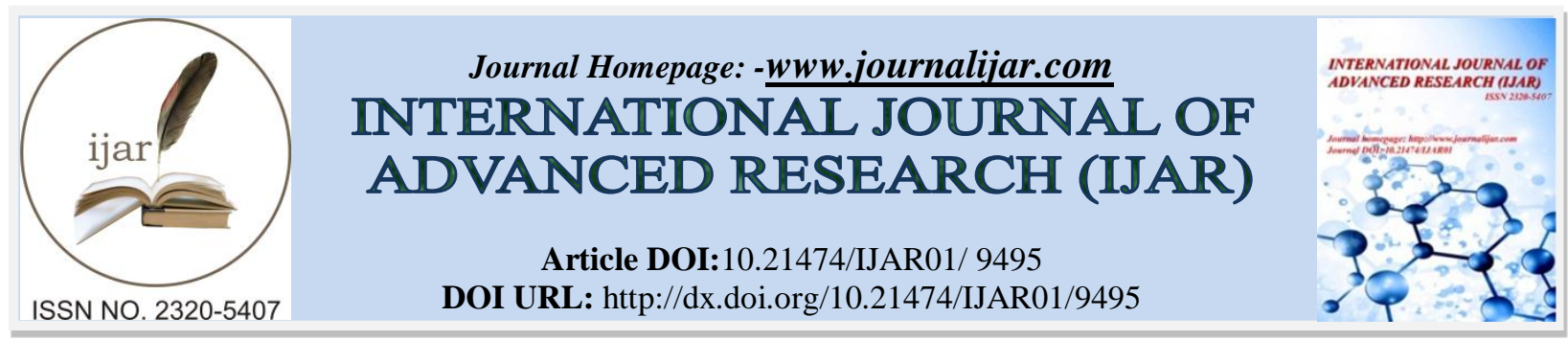

RESEARCH ARTICLE

\title{
EFFECTIVENESS OF COMMUNITY BASED NUTRITIONAL PROGRAMME ( CBNP) ON PREVENTION OF STUNTED GROWTH AMONG CHILDREN IN TERMS OF KNOWLEDGE AND EXPRESSED PRACTICES OF MOTHERS IN SELECTED VILLAGES OF AMBALA DISTRICT.
}

Ms. Kanika Kuhar ${ }^{1}$, Ms. Uma J. Deaver ${ }^{2}$, Ms. Amoldeep ${ }^{3}$ and Dr. Jyoti Sarin ${ }^{4}$.

1. M.Sc. Nursing Final Year Student, Department of Community Health Nursing, M.M College of Nursing, Maharishi Markandeshwar (Deemed to be University), Ambala, Haryana, India.

2. Professor Department of Community Health Nursing, M.M. College of Nursing, Maharishi Markandeshwar (Deemed to be University), Ambala, Haryana, India.

3. Assistant Professor Department of Community Health Nursing, M.M College of Nursing, Maharishi Markandeshwar (Deemed to be University), Ambala, Haryana, India.

4. Principal, M.M College of Nursing, Maharishi Markandeshwar (Deemed to be University), Ambala, Haryana, India.

\section{Manuscript Info}

\section{Manuscript History}

Received: 05 June 2019

Final Accepted: 07 July 2019

Published: August 2019

Key words:-

Community Based Nutritional Program (CBNP), Stunted growth, Knowledge, Expressed practice, mothers of under five children

\section{Abstract}

Stunting, or being too short for one's age, can cause physical, mental distress. The aim of the study was to assess the effectiveness of CBNP in terms of knowledge and expressed practice of mothers. A Quasi experimental pretest posttest design was used. Forty mothers were selected using purposive sampling technique. Structured Knowledge Questionnaire was used to assess knowledge and structured expressed practices rating scale was used to assess expressed practices of mothers. The study showed that mean posttest knowledge (14.2) and expressed practice score was (59.5) was higher than mean pretest knowledge (7.16) and expressed score was (49.3) respectively. There was significant association of pretest knowledge score with socioeconomic status and "ever taken any food preparing advice" and for expressed practices score with mother's education, occupation at 0.05 level of significance. The study concluded that CBNP can be used for improving the knowledge and expressed practices of mothers on prevention of stunted growth among children.

Copy Right, IJAR, 2019,. All rights reserved.

\section{Introduction:-}

Childhood stunting is one of the most significant impediments, something that makes it difficult to progress to human development. Stunting, or being too short for one's age, is defined as "height for age" value less than two standard deviations of the World Health Organization (WHO) child growth standards median. ${ }^{[1]}$

Globally approximately 165 million children under the age of 5 years are affected with stunting. Annual Global Nutrition report 2017 says $38.4 \%$ of children under age 5 were affected by stunting. The prevalence of stunting is higher in South Asian region. According to UNICEF; India is at the 10th spot among countries with the highest number of underweight children, and at the 17 th spot for the highest number of stunted children in the world. ${ }^{[2]}$ 
Stunted growth is not caused by single factor. Various factors are responsible for stunted growth. These can be classifies as knowledge of mother regarding breastfeeding, complementary and supplementary feeding, deworming, inadequate care of child, low socioeconomic status, unhealthy household environment, and lack of utilization of health care services. ${ }^{[3]}$ Nutrition-specific intervention would reduce stunting by about $20 \%$. A combination of nutrition -specific and nutrition-sensitive intervention appears necessary in order to optimize reductions in stunting. [4]

A cluster randomized trial on effect of de-worming on malnourished children was done for 2 years in which 5 round of Tab. Albendazole was given to preschoolers. They reported that there was height gain and more weight gain in the children after administration of Tab Albendazole. ${ }^{[5]}$

A study was conducted in human nutrition unit of All India Institute of Medical Sciences, New Delhi. Severely malnourished children 26, weight for age 55.27 \pm 3.14 , were identified in a colony of Muslim rural slum dwellers of low economic status. An equal number of normally nourished children matched for age, sex and per capita income were identified.A strong relation was found between nutritional status of the subjects and educational level of their mothers .It is concluded that maternal education and knowledge, attitude, practice are significantly and independently associated with children's nutritional status. ${ }^{[5]}$

Since mother's plays a prime role in providing nutrition. So they should have adequate knowledge of significance of protein and other nutrients in diet. So that mother can provide nutritious diet to their children which combat stunted growth.

\section{Methodology:-}

Quantitative research approach was adopted for the study and the design was "Quasi experimental: One group Pretest Post-test Design. The independent variable was Community based nutritional programme on prevention of stunted growth and the dependent variable were knowledge and expressed practices of mothers regarding prevention of stunted growth .

\section{Sampling Criteria: Following mothers were included in the study those:-}

1. having under five children

2. willing to participate in the study.

3. able to speak and understand Hindi

A quasi experimental design was taken up and 40 mothers were selected by purposive sampling technique. Sample attrition was 3 The reliability coefficient for structured knowledge questionnaire and expressed practice rating scale were calculated by KR-20 and Cronbach alpha method and it was found to be 0.76 and 0.87 respectively.

Ethical approval was obtained from the ethical committee of Maharishi Markandeshwar (Deemed to be University) Mullana, Ambala to conduct the final study. Permission for pilot study was taken from the Surpanch of Suhana village, Ambala. Permission for final study was taken from the Surpanch of Simbla and Mullana village ,Ambala.Purpose of the study was explained to sample subjects before data collection.

Selected Variables was used to collect data and to assess previous knowledge and expressed practices regarding prevention of stunted growth by using structured knowledge questionnaire and structured expressed practice rating scale respectively. Pre test was taken on first day and post test was taken on $15^{\text {th }}$ day. Structured Knowledge Questionnaire consisted of 20 multiple choice questions which are further categorized into four levels, very good $(>75 \%)$, good $(50-75 \%)$, average $(25-50 \%)$, below average $(\leq 25)$.

Structured Expressed Practice Rating Scale consisted of 26 questions. They are further categorized into three levels, inadequate practice $(<50 \%)$, moderate practice $(51-75 \%)$, adequate practice $(>75 \%)$

\section{Development of tools}

The tools were developed after reviewing the literature, seeking opinion from the experts. The content validity of the tools was established by 7 experts. Performa for selected variables consists of several items. Structured Knowledge Questionnaire to assess knowledge and expressed practices rating scale to assess expressed practices on prevention of stunted growth 
The final data was collected from October to November 2018 using structured knowledge questionnaire and expressed practices rating scale. Formal approval was taken from Sarpanch of Simbla and Mullana village. Written consent was taken from the subjects. On day $1^{\text {st }}$, pre assessment of knowledge and expressed practices was done by structured knowledge questionnaire and expressed practices rating scale and CBNP was given to each group of mothers on 2nd and 3rd day. On day 2nd session-1of CBNP health education was given to each group of five mothers for 90 minutes. On day 3rd session-2 of CBNP Mix veg. Khichdi/Dalia was demonstrated to each group of five mothers for 90 minutes. On day 4th session-3 of CBNP Mix veg Pratha/ORS/Handwashing was demonstrated to each group of five mothers for 90 minutes. On day 5th session-4 of CBNP Nutritional Barfi, nutritional ladoo and Panjeri was demonstrated to each group of five mothers for 90 minutes. On the 15 th day, post-test of knowledge and expressed practice on prevention of stunted growth was conducted.

\section{Statistical Analysis}

Data was analysed using Statistical Package for Social Sciences (SPSS) version 20. Data analysis was done by using descriptive statistics i.e. mean, standard deviation and inferential statistics such as t-test, ANOVA. Pearson "s correlation coefficient was used to check the relationship between the variable. Level of significance for the present study was $\mathrm{p} \leq 0.05$.

\section{Result:-}

\section{Section I: Selected Variables}

Level of significance Frequency and percentage distribution of mothers showed that out of 37 mothers, Out of 37 mothers, more than half of mothers $59.5 \%$ were in the age group of 26-30 years. $100 \%$ mothers belonged to Hindu religion. Less than half $45.9 \%$ were in category III and IV of socio economic status. Most of them51.4\% had completed secondary education .Majority 81.1\% mothers were housewife. Most of them 89.2\%,97.3\% mothers were living in pucca houses and own houses respectively. More than half $54.1 \%$ mothers monthly expenditure on food was $>30 \%$ of total income. More than half $70.3 \%$ mothers were vegetarian. $85.7 \%$ mothers had taken food preparing advice. Most of them 33.3\% had taken advice from mother in law. $100 \%$ mothers heard about prevention of stunted growth and Most of them $40.5 \%$ had source of information from neighborhood.

Frequency and percentage distribution of child according to selected variables. Out of 42 children, Less than half of children $42.9 \%$ were in the age group of 2-3 years. Majority $47.6 \%$ of children were in 1 st birth order. More than half $52.4 \%$ were girl. Less than half $40.5 \%$ children had attended play school education. Majority $83.3 \%, 90.5$ children were not in the risk of stunted according to weight for age and height for age. Majority85.7\% children had breastfeed exclusively for 6 months. All children had taken supplementary food at 6 month of age. Majority $90.5 \%$ children were immunized. Majority $95.2 \%$ children had taken deworming tablets. Most of $78.6 \%$ children were suffered from diarrheal disease during the last 7 days. Out of $21.4 \%$ children, $14.3 \%$ were treated by home remedies and $21.4 \%$ children were recovered with in less than 7 day. Half of the children $50 \%$ were suffered from any other infection in the last 7 days. Out of $50 \%$ children $23.9 \%$ were suffering from respiratory infection.

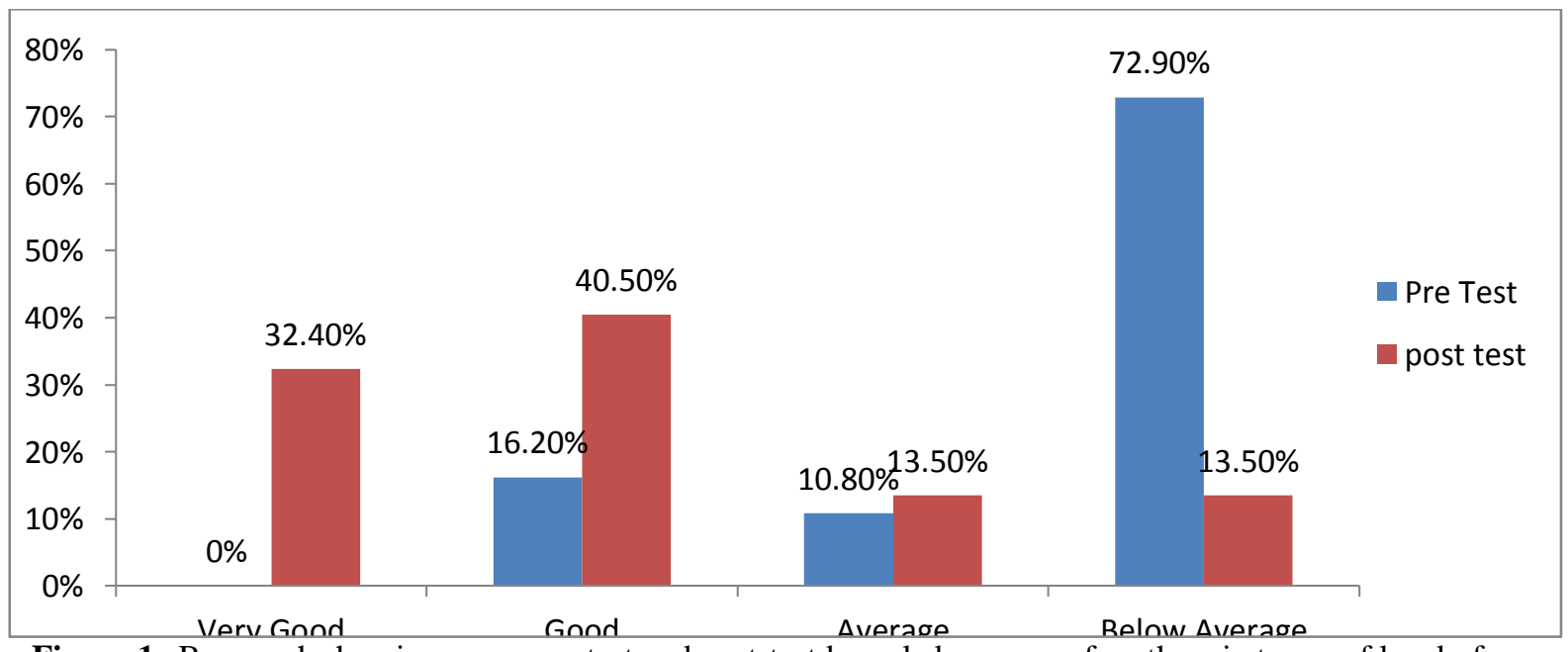

Figure 1:-Bar graph showing mean pre-test and post-test knowledge score of mothers in terms of level of knowledge on prevention of stunted growth among children 
Figure 1 shows the frequency and percentage distribution of mothers in terms of level of knowledge. In pre-test of structured knowledge questionnaire most of the mothers $72.9 \%$ had below average, $10.8 \%$ had average knowledge and only $16.2 \%$ had good knowledge whereas in post-test nearly one third $32.4 \%$ of mothers had very good knowledge, less than half $40.5 \%$ of mothers had good knowledge ,only $13.5 \%$ of them had average knowledge and below average knowledge on prevention of stunted growth among children

Table 1:-Range, Mean, Standard Deviation and Median of Pre-Test and Post-Test Knowledge Score of mothers on prevention of stunted growth among children.

$\mathbf{N}=\mathbf{3 7}$

\begin{tabular}{|r|r|r|r|}
\hline Knowledge Test & Range & Mean \pm S.D. & Median \\
\hline Pre-Test & $1-15$ & $7.16 \pm 4.34$ & 6.00 \\
Post-Test & $6-19$ & $14.24 \pm 3.23$ & 15.00 \\
\hline
\end{tabular}

Minimum Score $=0$

Maximum Score $=20$

Table 1 shows that mean post test knowledge score $(14.24+3.2)$ was higher than mean pre-test knowledge score $(7.16+4.34)$. The median for post test was 15.0 and for pre test was 6.0 These findings showed that mothers developed good knowledge on prevention of stunted growth after the CBNP.

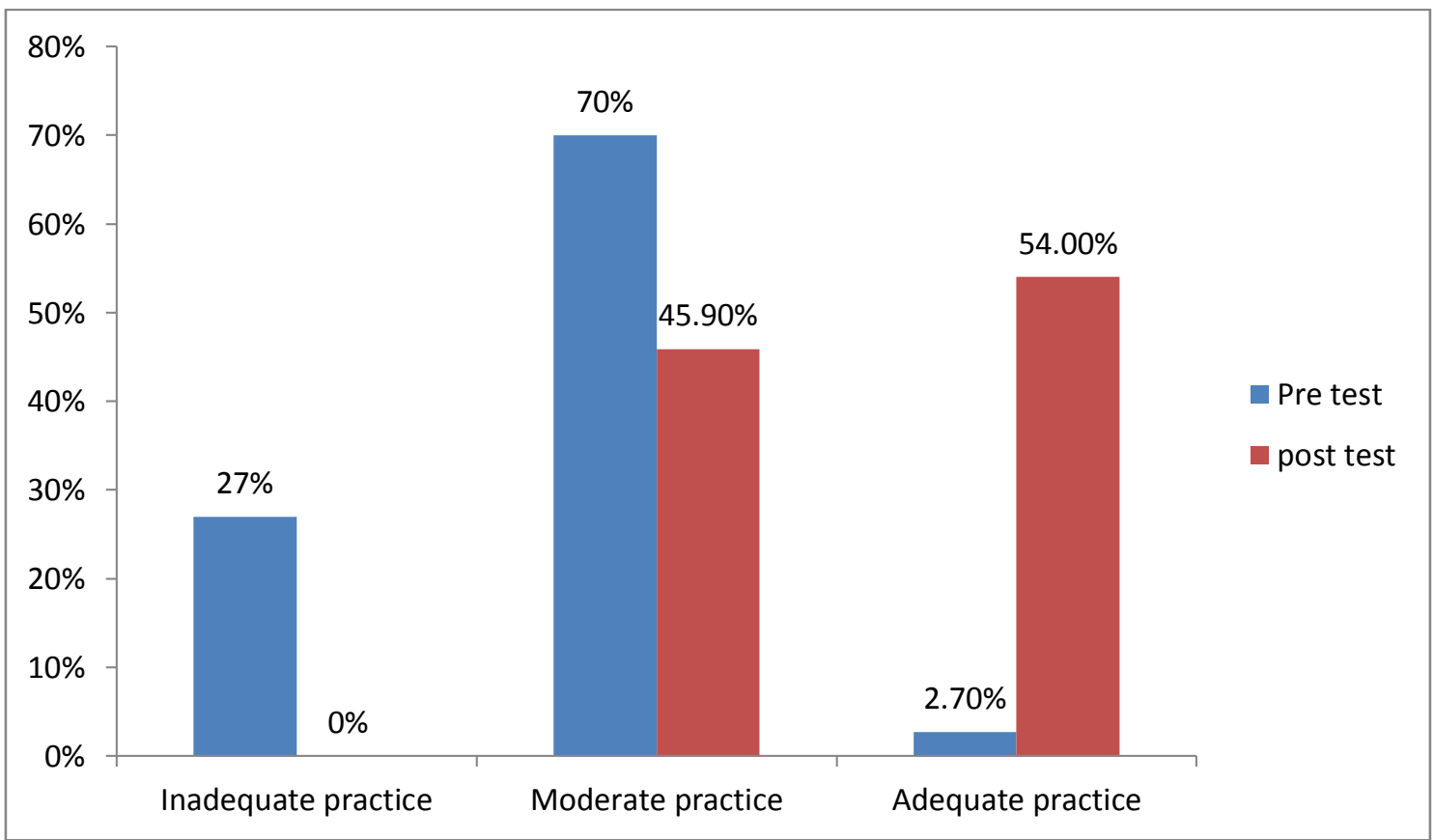

Figure 2:-Bar graph showing mean pre-test and post-test of mothers in terms of level of expressed practice on prevention of stunted growth among children

Figure 2 shows the overall frequency and percentage distribution of mothers in terms of level of expressed practice. In pre-test of most of mothers $70.0 \%$ had moderate practice, only $2.7 \%$ had adequate practices. Whereas in posttest more than half of mothers $54.0 \%$ had adequate practice, less than half $45.9 \%$ had moderate practices. .

Table 2:-Range, Mean, Standard Deviation and Median of Pre-Test and Post-Test Expressed Practice Score of Mothers on Prevention of Stunted growth among children.

\begin{tabular}{|l|l|l|l|}
\hline Practice Test & Range & Mean \pm S.D. & Median \\
\hline Pre-Test & $53-58$ & $49.30+1.15$ & 51.00 \\
Post-Test & $57-64$ & $59.57+0.51$ & 60.00 \\
\hline
\end{tabular}

Minimum Score $=26$

Maximum Score $=78$ 
Table 2 shows that mean post test expressed practice score $(59.57+0.51)$ was higher than mean pre-test practice score $(49.30+1.15)$. The median for post test was 60.0 and for pre test was 51.0. These findings show that mothers improved in there practices as expressed by them.

Table 3:-Correlation between Post-Test Knowledge and Expressed Practice Scores of Mothers on Prevention of Stunted growth among children.

\begin{tabular}{|l|c|c|}
\hline Pre-Test & $\begin{array}{c}\text { Expressed Practice score } \\
\text { r (p value) }\end{array}$ & Knowledge score \\
\hline Knowledge score & $0.70\left(0.00^{*}\right)$ & - \\
\hline
\end{tabular}

df- 36, $\mathbf{r}(36)=0.32$

*Significance $(\mathbf{p}<0.05)$

Table 3.1 depicts the correlation between knowledge and expressed practice score of mothers. It shows that ' $r$ ' value 0.32 which was found to be statistically significant between pre -test knowledge and expressed practice score. It shows there was highly positive co-relation between knowledge and expressed practice scores.

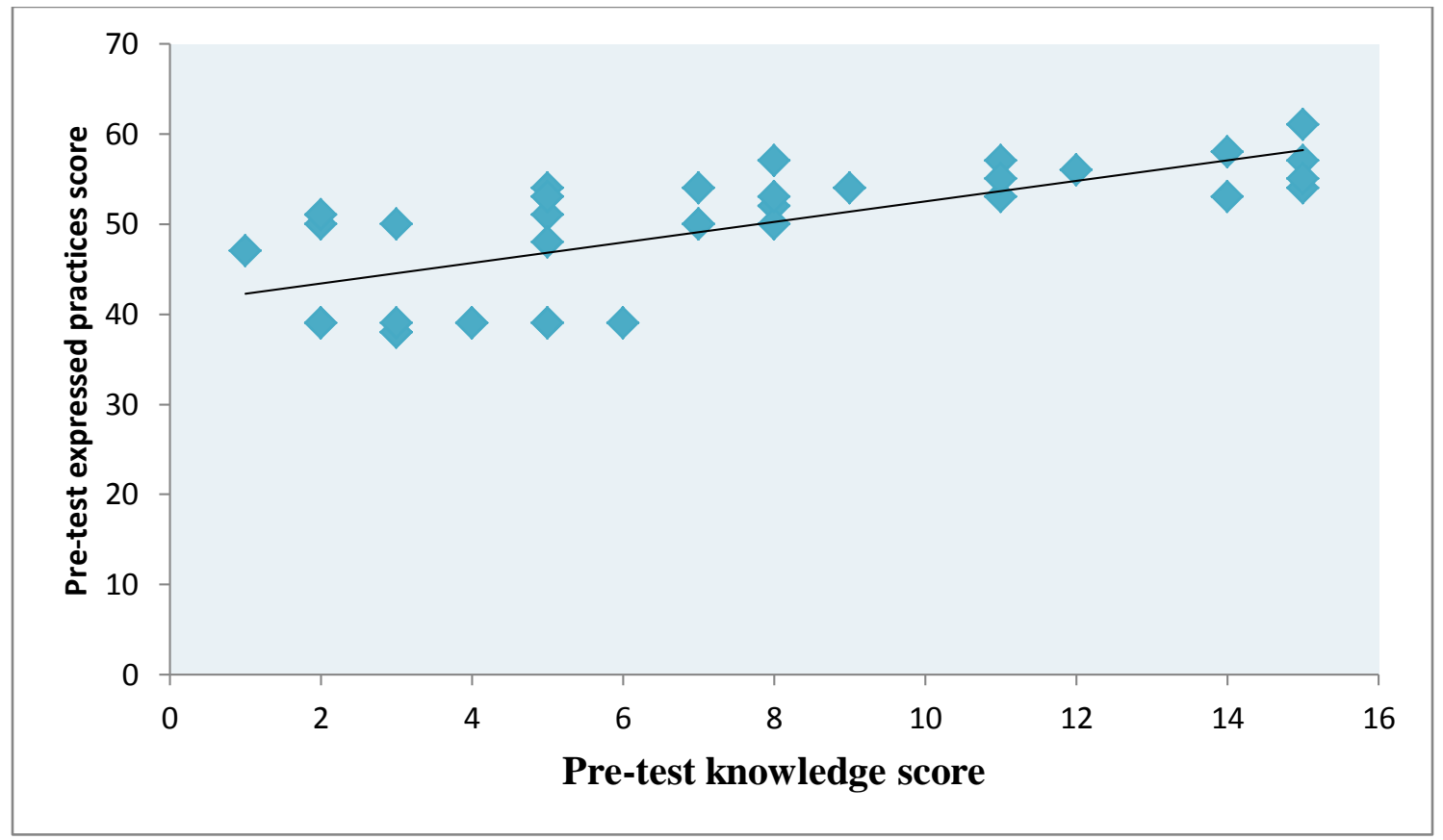

Figure 3:-Scatter diagram showing Correlation between Pre -Test knowledge and expressed practice scores of mothers on prevention of stunted growth among children.

Table 3:-Correlation between Post-Test Knowledge and Expressed Practice Scores of Mothers on Prevention of Stunted growth among children.

\begin{tabular}{|l|c|c|}
\hline Post-Test & $\begin{array}{c}\text { Expressed Practice score } \\
\text { r (p value) }\end{array}$ & Knowledge score \\
\hline Knowledge score & $0.72\left(0.00^{*}\right)$ & - \\
\hline
\end{tabular}

df- 36, $\mathbf{r}(36)=0.32$

*Significance $(\mathbf{p}<0.05)$

Table 3 depicts the correlation between knowledge and expressed practice score of mothers. It shows that ' $r$ ' value 0.32 which was found to be statistically significant between post-test knowledge and expressed practice score. It shows there was highly positive co-relation between knowledge and expressed practice scores. 


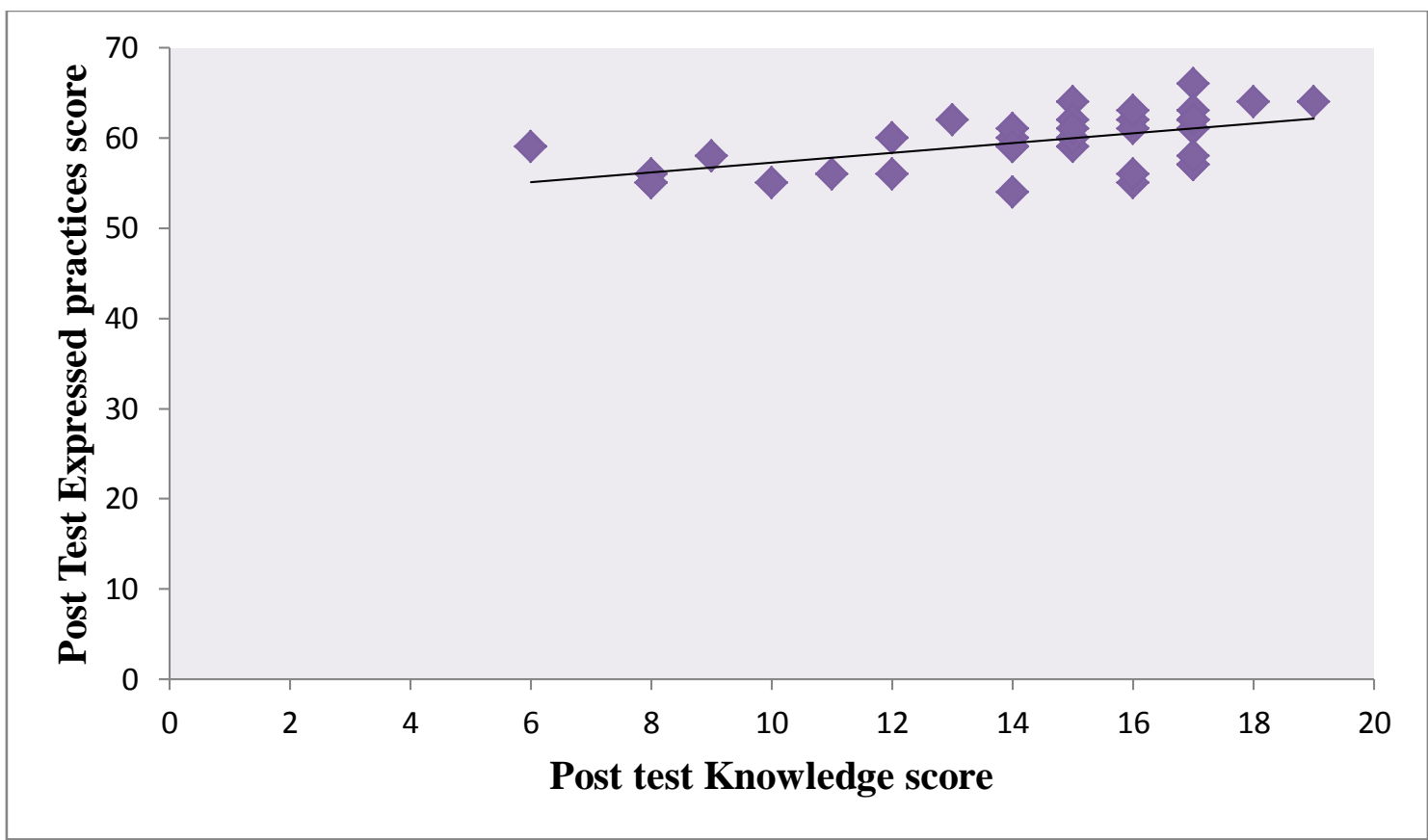

Figure 3:-Scatter diagram showing Correlation between Post-test knowledge and expressed practice scores of mothers on prevention of stunted growth among children.

Table 4:-ANOVA and t- Value Showing Association of Mean Pre-Test Knowledge Score of Mothers on Prevention of Stunted growth among Children With Selected Variables.

\begin{tabular}{|c|c|c|c|c|}
\hline & & \multicolumn{3}{|c|}{$\mathbf{N}=\mathbf{3 7}$} \\
\hline Selected variables & Mean & $\mathbf{f} / \mathbf{t}$ & df & p value \\
\hline \multicolumn{5}{|l|}{ 1. Age } \\
\hline 1.1) 20-25 year & 7.88 & 0.17 & $3 / 33$ & $0.91^{\mathrm{NS}}$ \\
\hline 1.2)26-30 year & 6.91 & & & \\
\hline 1.3)31-35 year & 7.50 & & & \\
\hline 1.4)36-40 year & 5.00 & & & \\
\hline \multicolumn{5}{|l|}{ 2.Type of family } \\
\hline 2.1) Joint & 6.77 & 0.75 & $2 / 34$ & $0.48^{\mathrm{NS}}$ \\
\hline 2.2) Nuclear & 6.78 & & & \\
\hline 2.3) Extended & 9.17 & & & \\
\hline \multicolumn{5}{|l|}{$\begin{array}{l}\text { 3.Total Monthly Income of the } \\
\text { Family }\end{array}$} \\
\hline 3.1) Rs $>126,360$ & - & 4.67 & $3 / 33$ & \multirow[t]{7}{*}{$0.00^{*}$} \\
\hline 3.2) Rs $63,182-126,356$ & - & & & \\
\hline 3.3) Rs 47,266-63178 & 10.5 & & & \\
\hline 3.4) Rs 31,591-47262 & 9.64 & & & \\
\hline 3.5) Rs 18,953-31589 & 7.55 & & & \\
\hline 3.6) Rs 6327-18949 & 4.23 & & & \\
\hline 3.7) $\mathrm{Rs} \leq 6323$ & - & & & \\
\hline \multicolumn{5}{|l|}{ 4.Socioeconomic status } \\
\hline 4.1)Category (I) & - & & & \\
\hline 4.2)Category (II) & 11.00 & 6.177 & $3 / 33$ & $0.00 *$ \\
\hline 4.3)Category (III) & 9.47 & & & \\
\hline 4.4)Category (IV) & 4.67 & & & \\
\hline 4.5)Category (V) & 3.00 & & & \\
\hline 5.1) Non literate & 3.00 & \multirow[t]{2}{*}{2.28} & \multirow[t]{2}{*}{$3 / 32$} & \multirow[t]{2}{*}{$0.08^{\mathrm{NS}}$} \\
\hline 5.2) primary & 5.00 & & & \\
\hline
\end{tabular}




\begin{tabular}{|c|c|c|c|c|}
\hline 5.3) secondary & 5.89 & & & \\
\hline 5.4) graduate & 10.14 & & & \\
\hline 5.5) post graduate & 9.14 & & & \\
\hline 6.Mother's occupation & & \multirow{6}{*}{5.58} & \multirow{6}{*}{$4 / 32$} & \multirow{6}{*}{$0.00 *$} \\
\hline 6.1) House wife & 5.93 & & & \\
\hline 6.2) Public / Medical & 14.00 & & & \\
\hline 6.3) Private / Medical & 14.50 & & & \\
\hline 6.4) Public / Non -medical & 15.00 & & & \\
\hline 6.5) Private / Non - medical & 9.67 & & & \\
\hline \multicolumn{5}{|l|}{ 7.Type of house } \\
\hline 7.1) Pucca & 7.24 & 0.31 & 35 & $0.87^{\mathrm{NS}}$ \\
\hline 7.2) Kuccha & 6.50 & & & \\
\hline \multicolumn{5}{|l|}{ 8.Monthly expenditure on food } \\
\hline \multicolumn{5}{|l|}{ 8.1)Less than $10 \%$} \\
\hline 8.2$) 10 \%-30 \%$ of total income & 8.00 & 1.17 & $1 / 35$ & $0.28^{\mathrm{NS}}$ \\
\hline 8.3$)>30 \%$ of total income & 6.45 & & & \\
\hline \multicolumn{5}{|l|}{ 9.Dietary history } \\
\hline 9.1) Vegetarian & 7.00 & 0.86 & $2 / 34$ & $0.43^{\mathrm{NS}}$ \\
\hline 9.2) Non-Vegetarian & 6.29 & & & \\
\hline 9.3) Eggetarian & 9.75 & & & \\
\hline \multicolumn{5}{|l|}{$\begin{array}{l}\text { 10.Have you ever taken any food } \\
\text { preparing advice? }\end{array}$} \\
\hline $10.1 \mathrm{Yes}$ & 7.52 & 1.43 & 35 & $0.02 *$ \\
\hline $10.2 \mathrm{No}$ & 4.25 & & & \\
\hline \multicolumn{5}{|l|}{ If yes specify $(\mathrm{N}=36)$} \\
\hline 10.1.1Mother in law & 6.85 & & & \\
\hline 10.1.2Sister & 6.67 & & & \\
\hline 10.1.3 Grandmother & 12.50 & 1.96 & $5 / 30$ & $0.10^{\mathrm{NS}}$ \\
\hline 10.1.4Sister in law & 2.00 & & & \\
\hline 10.1.5Neighbour & 15.00 & & & \\
\hline 10.1.6Other & 8.00 & & & \\
\hline \multicolumn{5}{|l|}{$\begin{array}{l}\text { If yes, source of information about } \\
\text { stunted growth }(\mathrm{N}=41)\end{array}$} \\
\hline 11.1) Media & 6.25 & & & \\
\hline 11.2.)Health Personnel & 6.33 & 1.27 & $4 / 36$ & $0.29^{\mathrm{NS}}$ \\
\hline 11.3 Newspaper & 9.33 & & & \\
\hline 11.4) Neighbour & 6.23 & & & \\
\hline 11.5 )Any specific & 3.00 & & & \\
\hline
\end{tabular}

Sample characterstics of child

\begin{tabular}{|c|c|c|c|c|}
\hline \multicolumn{3}{|c|}{ Sample characterstics of child } & \multicolumn{2}{|c|}{$\mathbf{N}=42$} \\
\hline \multicolumn{3}{|c|}{ 1.Age of the child } & & \\
\hline 1.1) $1-2$ year & 6.33 & & & \\
\hline 1.2) 2-3 year & 8.67 & & & \\
\hline 1.3) 3-4 year & 7.14 & 0.69 & $5 / 36$ & $0.63^{\mathrm{NS}}$ \\
\hline 1.4) 4-5 year & 6.32 & & & \\
\hline \multicolumn{5}{|l|}{ 2.Order of child } \\
\hline 2.2) 1 & 7.36 & & & \\
\hline 2.2) 2 & 7.11 & 0.03 & $3 / 38$ & $0.99^{\mathrm{NS}}$ \\
\hline 2.3) 3 & 7.00 & & & \\
\hline 2.4) 4 & 6.00 & & & \\
\hline \multicolumn{5}{|l|}{ 3.Gender } \\
\hline 3.1Boy & 6.59 & 7.35 & 35 & $0.28^{\mathrm{NS}}$ \\
\hline 3.2Girl & 7.65 & & & \\
\hline
\end{tabular}




\begin{tabular}{|c|c|c|c|c|}
\hline \multicolumn{5}{|l|}{ 4.Education status } \\
\hline 4.1 Attending Aaganwadi & 6.81 & & & \\
\hline 4.2 Attending play school & 6.38 & 1.07 & $2 / 39$ & $0.35^{\mathrm{NS}}$ \\
\hline 4.3 Not to any & 9.13 & & & \\
\hline \multicolumn{5}{|l|}{ 5.Weight for age } \\
\hline $\begin{array}{l}\text { 5.1) Severe malnutrition }=<- \\
\text { 3SD }\end{array}$ & 7.00 & & & \\
\hline $\begin{array}{l}\text { 5.2) Mild malnutrition=-3to- } \\
\text { 2SD }\end{array}$ & 9.00 & 0.62 & $2 / 38$ & $0.54^{\mathrm{NS}}$ \\
\hline 5.3)Normal $=-2$ to $+2 \mathrm{SD}$ & $6 . .80$ & & & \\
\hline 5.4) obesity=>-2SD & - & & & \\
\hline \multicolumn{5}{|l|}{ 6.Height for age } \\
\hline 6.1)Normal $=-2$ to $+2 \mathrm{SD}$ & 7.30 & & & \\
\hline 6.2)Stunted $=<-2 \mathrm{SD}$ & 4.33 & 1.04 & $2 / 39$ & $0.36^{\mathrm{NS}}$ \\
\hline 6.3)Severe stunted $=<-3 \mathrm{SD}$ & 11.00 & & & \\
\hline \multicolumn{5}{|l|}{$\begin{array}{l}\text { 7.Have you breast feed the baby } \\
\text { exclusively for } 6 \text { months? }\end{array}$} \\
\hline 7.1 ) Yes & 6.97 & 0.50 & 35 & $0.99^{\mathrm{NS}}$ \\
\hline 7.2) No & 8.40 & & & \\
\hline \multicolumn{5}{|l|}{ 8. Was the child immunized? } \\
\hline 8.1)Completely & 7.11 & & & \\
\hline 8.2) Partially & 8.00 & 0.27 & 35 & $0.72^{\mathrm{NS}}$ \\
\hline \multicolumn{5}{|l|}{$\begin{array}{l}\text { 9. Was you given deworming tablets } \\
\text { to your child? }\end{array}$} \\
\hline 9.1) Yes & 8.00 & - & - & - \\
\hline 9.2) No & 7.14 & & & \\
\hline $\begin{array}{l}\text { If yes when did you last deworm } \\
\text { your child? }(\mathrm{N}=40) \\
\text { 9.2.1) } 1-3 \text { month back }\end{array}$ & 6.91 & & & \\
\hline 9.2.2)4-6 month back & $8 . .75$ & & & \\
\hline 9.2.3)7-9 month back & 9.00 & 0.38 & $4 / 35$ & $0.81^{\mathrm{NS}}$ \\
\hline 9.2.4)10-12 month back & 6.40 & & & \\
\hline 9.2.5) Don’t know & 6.62 & & & \\
\hline \multicolumn{5}{|l|}{$\begin{array}{l}\text { 10.Has the child suffered from } \\
\text { diarrheal disease during the last } 7 \\
\text { days? }\end{array}$} \\
\hline 10.1 YES & 6.4 & 0.56 & 35 & $0.98^{\mathrm{NS}}$ \\
\hline $10.2 \mathrm{NO}$ & 7.3 & & & \\
\hline \multicolumn{5}{|l|}{$\begin{array}{l}\text { If yes ,how did you treat your child } \\
\text { with diarrhea? }(\mathrm{N}=9)\end{array}$} \\
\hline 10.1.1) hospital & 7.00 & 0.48 & 7 & $0.19^{\mathrm{NS}}$ \\
\hline 10.1.2) home treatment & 5.33 & & & \\
\hline \multicolumn{5}{|l|}{$\begin{array}{l}\text { 11. Was the child suffered from any } \\
\text { other illness in the last } 7 \text { days? }\end{array}$} \\
\hline 11.1 YES & 7.89 & 0.98 & 35 & $0.72^{\mathrm{NS}}$ \\
\hline $11.2 \mathrm{NO}$ & 6.47 & & & \\
\hline \multicolumn{5}{|l|}{ 12.1)If yes specify $(\mathrm{N}=21)$} \\
\hline 12.1.1)Respiratory infection & 6.91 & & & \\
\hline 12.1.2)Gastrointestinal infection & 7.14 & 1.41 & $7 / 29$ & $0.23^{\mathrm{NS}}$ \\
\hline 12.1.3)Fever & 10.86 & & & \\
\hline \multicolumn{5}{|l|}{$\begin{array}{l}\text { Within how many days the child } \\
\text { recovered } ?(\mathrm{~N}=21)\end{array}$} \\
\hline Less than seven day & 7.58 & & & \\
\hline More than seven day & 8.80 & 0.52 & 19 & $0.32^{\mathrm{NS}}$ \\
\hline
\end{tabular}


Table 4. depicts the ANOVA/' $t$ ' value of age (0.91), type of family (0.48), occupation of the head of the family(0.06), education of the head of the family (0.19), mother's education $(0.08)$,type of house $(0.87)$, monthly expenditure on food $(0.28)$, dietary history $(0.43 \%)$, have you ever taken any food preparing advice $(0.10)$,source of information about prevention of stunted growth(0.29), age of the child (0.63), order of child(0.99),gender (0.28), education status(0.35), weight for age (0.54), height for age (0.36), have you breast feed the baby exclusively for 6 months(0.99), is the child immunized (0.72), have you given deworming tablets to your child (0.81), Has the child suffered from diarrheal disease during the last 7 days(0.98), has the child suffered from any other illness in the last 7 days (0.72), Within how many days the child recovered (0.32) were found to be non significant. Thus knowledge score of mothers were independent of selected variable whereas computed ANOVA value of total monthly income of the family $\left(0.00^{*}\right)$, socioeconomic status $\left(0.00^{*}\right)$, mother's occupation $\left(0.00^{*}\right)$, ever taken any food preparing advice $\left(0.02^{*}\right)$.was found to be statistically significant at 0.05 level of significance. It denotes the association with knowledge.

Table 4:-ANOVA and t- Value Showing Association of Level of Mean Post-Test Expressed Practice Score With Selected Variables.

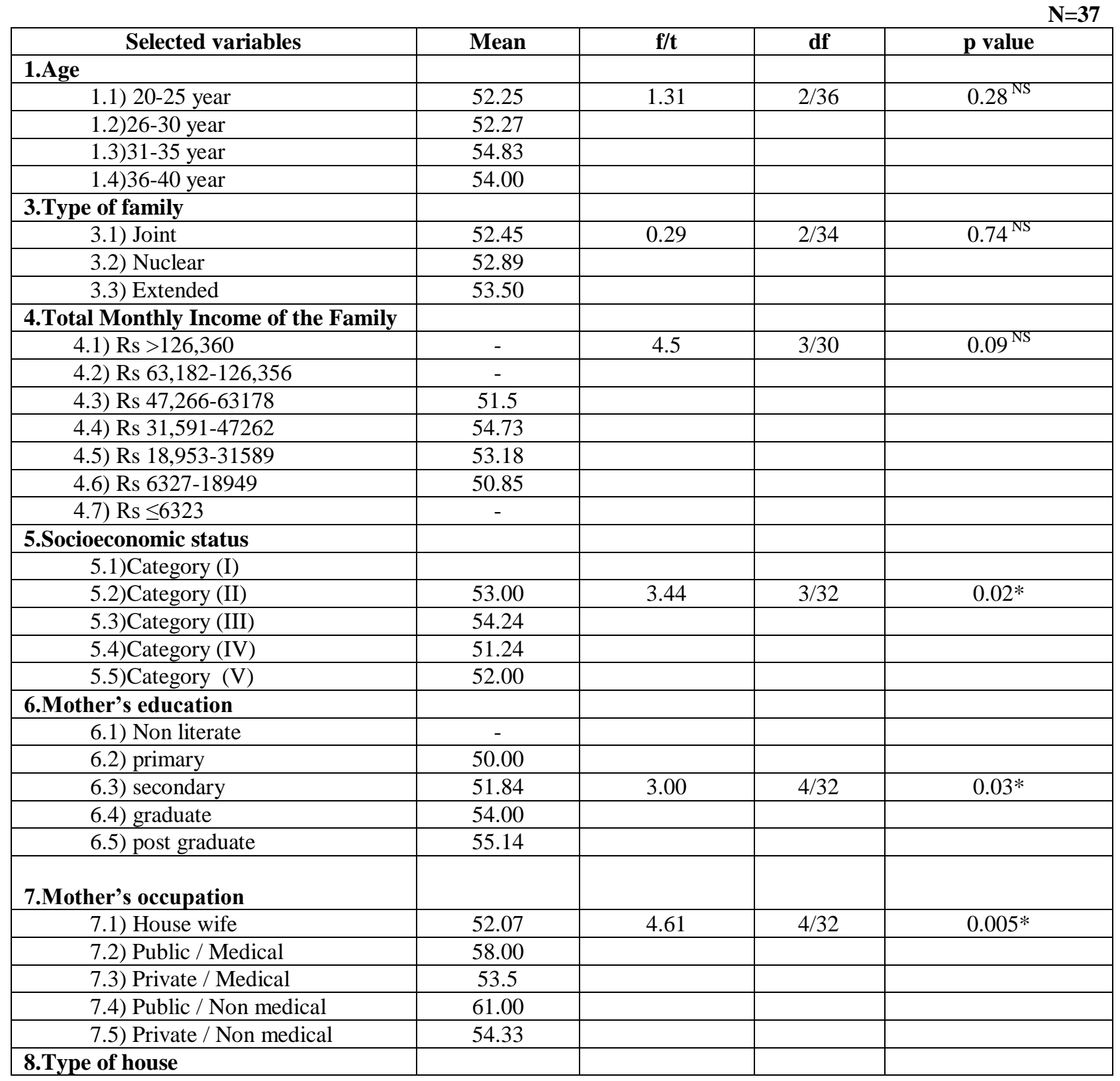




\begin{tabular}{|c|c|c|c|c|}
\hline 8.1) Pucca & 52.94 & 1.23 & 35 & $0.22^{\mathrm{NS}}$ \\
\hline 8.2) Kuccha & 51.00 & & & \\
\hline \multicolumn{5}{|l|}{ 9.Resident } \\
\hline 9.1) Owns & 52.75 & 0.24 & 35 & $0.80^{\mathrm{NS}}$ \\
\hline 9.2) Rented & 52.00 & & & \\
\hline \multicolumn{5}{|l|}{ 10.Monthly expenditure on food } \\
\hline \multicolumn{5}{|l|}{ 10.1)Less than $10 \%$} \\
\hline 10.2$) 10 \%-30 \%$ of total income & 53.18 & 0.69 & $1 / 34$ & $0.41^{\mathrm{NS}}$ \\
\hline 10.3$)>30 \%$ of total income & 52.35 & & & \\
\hline \multicolumn{2}{|l|}{ 11.Dietary history } & \multirow[t]{4}{*}{1.1} & \multirow[t]{4}{*}{$2 / 34$} & \multirow[t]{4}{*}{$0.21^{\mathrm{NS}}$} \\
\hline 11.1) Vegetarian & 52.65 & & & \\
\hline 11.2) Non-Vegetarian & 51.71 & & & \\
\hline 11.3) Eggetarian & 55.00 & & & \\
\hline \multicolumn{5}{|l|}{$\begin{array}{l}\text { 12.Have you ever taken any food } \\
\text { preparing advice? }\end{array}$} \\
\hline 12.1 Yes & 49.79 & 1.22 & 40 & $0.56^{\mathrm{NS}}$ \\
\hline $12.2 \mathrm{No}$ & 45.25 & & & \\
\hline \multicolumn{5}{|l|}{ If yes specify $(\mathrm{N}=36)$} \\
\hline 12.1.1Mother in law & 49.31 & & & \\
\hline 12.1.2Sister & 48.67 & & & \\
\hline 12.1.3 Grandmother & 53.00 & 0.28 & $5 / 30$ & $0.94^{\mathrm{NS}}$ \\
\hline 12.1.4Sister in law & 50.00 & & & \\
\hline 12.1.5Neighbour & 55.00 & & & \\
\hline 12.1.6 Other & 49.56 & & & \\
\hline \multicolumn{5}{|l|}{$\begin{array}{l}\text { 13.If yes, source of information } \\
(\mathrm{N}=41)\end{array}$} \\
\hline 13.1) Media & 48.00 & & & \\
\hline 13.2.)Health Personnel & 48.67 & 1.81 & $4 / 36$ & $0.15^{\mathrm{NS}}$ \\
\hline 13.3 )Newspaper & 52.95 & & & \\
\hline 13.4) Neighbour & 47.77 & & & \\
\hline 13.5 )Any specific & 38.00 & & & \\
\hline
\end{tabular}

Sample characterstics of child

\begin{tabular}{|c|c|c|c|c|}
\hline & & & & \\
\hline 1.Age of the child & & & & \\
\hline 1.1) 1-2 year & 46.17 & & & \\
\hline 1.2) 2-3 year & 50.17 & & & \\
\hline 1.3) 3-4 year & 47.86 & 1.03 & $5 / 37$ & $0.41^{\mathrm{NS}}$ \\
\hline 1.4) 4-5 year & 46.89 & & & \\
\hline 2.Order of child & & & & \\
\hline 2.2) 1 & 49.36 & & & \\
\hline 2.2) 2 & 49.61 & 0.73 & $3 / 38$ & $0.54^{\mathrm{NS}}$ \\
\hline 2.3) 3 & 50.25 & & & \\
\hline 2.4) 4 & 39.00 & & & \\
\hline 3.Gender & & & & \\
\hline 3.1Boy & 49.0 & 0.18 & 40 & $0.45^{\mathrm{NS}}$ \\
\hline 3.2Girl & 49.50 & & & \\
\hline 4.Education status & & & & \\
\hline 4.1 Attending Aaganwadi & 49.38 & & & \\
\hline 4.2 Attending play school & 46.77 & 2.24 & $2 / 39$ & $0.12^{\mathrm{NS}}$ \\
\hline 4.3 Not to any & 53.25 & & & \\
\hline 5.Weight for age & & & & \\
\hline 5.1) Severe malnutrition=<-3SD & 50.0 & & & \\
\hline 5.2) Mild malnutrition=-3to- & 49.67 & 0.01 & $3 / 38$ & $0.98^{\mathrm{NS}}$ \\
\hline
\end{tabular}




\begin{tabular}{|c|c|c|c|c|}
\hline 2SD & & & & \\
\hline 5.3)Normal=-2 to $+2 \mathrm{SD}$ & 49.20 & & & \\
\hline 5.4) obesity=>-2SD & - & & & \\
\hline \multicolumn{5}{|l|}{ 6.Height for age } \\
\hline 6.1)Normal $=-2$ to +2 SD & 49.64 & & & \\
\hline 6.2) Stunted $=<-2 \mathrm{SD}$ & 43.67 & 1.35 & $2 / 39$ & $0.27^{\mathrm{NS}}$ \\
\hline 6.3)Severe stunted $=<-3 \mathrm{SD}$ & 55.00 & & & \\
\hline \multicolumn{5}{|l|}{$\begin{array}{l}\text { 7.Have you breast feed the baby } \\
\text { exclusively for } 6 \text { months? }\end{array}$} \\
\hline 7.1$)$ Yes & 48.66 & 1.42 & 40 & $0.007 *$ \\
\hline 7.2) No & 53.40 & & & \\
\hline \multicolumn{5}{|l|}{ 8. Was the child immunized? } \\
\hline 8.1)Completely & 49.03 & 0.97 & 40 & $0.06^{\mathrm{NS}}$ \\
\hline 8.2) Partially & 54.00 & & & \\
\hline \multicolumn{5}{|l|}{$\begin{array}{l}\text { 9.Have you given deworming tablets } \\
\text { to your child? }\end{array}$} \\
\hline 9.1) Yes & 53.00 & 0.52 & 40 & $0.60^{\mathrm{NS}}$ \\
\hline 9.2) No & 49.19 & & & \\
\hline $\begin{array}{l}\text { 10.If yes when did you last deworm } \\
\text { your child? }(\mathbf{N}=\mathbf{4 0}) \\
\text { 10.2.1) } 1-3 \text { month back }\end{array}$ & 50.73 & & & \\
\hline 10.2.2)4-6 month back & 51.00 & & & \\
\hline 10.2.3)7-9 month back & 55.00 & 1.69 & $4 / 35$ & $0.17^{\mathrm{NS}}$ \\
\hline 10.2.4) 10-12 month back & 44.60 & & & \\
\hline 10.2.5) Don't know & 47.62 & & & \\
\hline \multicolumn{5}{|l|}{$\begin{array}{l}\text { 11.Has the child suffered from } \\
\text { diarrheal disease during the last } 7 \\
\text { days? }\end{array}$} \\
\hline 11.1 YES & 50.78 & 0.72 & 40 & $0.08^{\mathrm{NS}}$ \\
\hline $11.2 \mathrm{NO}$ & 48.28 & & & \\
\hline \multicolumn{5}{|l|}{$\begin{array}{l}\text { If yes ,how did you treat your child } \\
\text { with diarrhea? }(\mathrm{N}=9)\end{array}$} \\
\hline 11.1.1) hospital & 51.83 & 0.81 & 7 & $0.04 *$ \\
\hline 11.1.2) home treatment & 48.67 & & & \\
\hline \multicolumn{5}{|l|}{$\begin{array}{l}\text { 12. Has the child suffered from any } \\
\text { other illness in the last } 7 \text { days? }\end{array}$} \\
\hline 12.1 YES & 50.11 & 0.67 & 40 & $0.33^{\mathrm{NS}}$ \\
\hline $12.2 \mathrm{NO}$ & 48.53 & & & \\
\hline \multicolumn{5}{|l|}{ If yes specify $(\mathrm{N}=21)$} \\
\hline 12.1. 1) Respiratory infection & 50.73 & & & \\
\hline 12.1.2) Gastrointestinal infection & 49.19 & 1.21 & $7 / 14$ & $0.32^{\mathrm{NS}}$ \\
\hline 12.1.3) Fever & 52.60 & & & \\
\hline \multicolumn{5}{|l|}{$\begin{array}{l}\text { Within how many days the child } \\
\text { recovered } ?(\mathrm{~N}=21)\end{array}$} \\
\hline 12.1.1.1)Less than seven day & 50.23 & & & \\
\hline 12.1.1.2)More than seven day & 49.80 & 0.12 & 19 & $0.16^{\mathrm{NS}}$ \\
\hline
\end{tabular}

Table 4 depicts the ANOVA/' $t$ ' value of age (0.28), type of family (0.74), occupation of the head of the family (0.06), education of the head of the family (0.36), total monthly income of the family (0.09), type of house $(0.22)$, resident $(0.80)$, monthly expenditure on food $(0.41)$, dietary history $(0.21)$, have you ever taken any food preparing advice (0.56), source of information about stunted growth (0.15), age of the child (0.41),order of child(0.54), gender (0.45), education status( 0.12 ), weight for age (0.98), height for age (0.27), is the child immunized (0.72), have you given deworming tablets to your child (0.60), Has the child suffered from diarrheal disease during the last 7 days(0.08), has the child suffered from any 
other illness in the last 7 days (0.33), Within how many days the child recovered (0.16) were found to be non significant. Thus expressed practice score of mothers were independent of selected variable whereas computed ANOVA value of socioeconomic $\operatorname{status}(0.02 *)$, mother's education $\left(0.03^{*}\right)$, mother's occupation $\left(0.00^{*}\right)$, have you breast feed the baby exclusively for 6 months $\left(0.00^{*}\right)$, how did you treat your child with diarrhea $\left(0.04^{*}\right)$ was found to be statistically significant at 0.05 level of significance. It denotes the association with expressed practice.

\section{Discussion:-}

In present study, More than half of mothers (59.5\%) belong to age group of 26-30 year.These findings are partialy consistent with the study conducted by Beniam Daniel where they found that less than half (34\%) belong to age group of 26-30 year. In the present study, None of the mothers had the very good knowledge regarding prevention of stunted growth and $72.9 \%$ had below average knowledge before the community based nutritional programme whereas $32.4 \%$ had very good knowledge while only $13.5 \%$ had below average knowledge regarding prevention of stunted growth after administration of community based nutritional programme.

Similar findings were reported in a study conducted by M.edith to evaluate the effectiveness of dietary practices to assess knowledge regarding prevention of malnutrition among under five children, where the findings revealed that majority $56 \%$ of mothers had moderately adequate knowledge and $22 \%$ of mothers had adequate knowledge.

In the present study, pre test and post test knowledge mean score was 7.16 and 14.24 respectively which shows an increase in knowledge score of mothers after administration of CBNP. The findings of study consistent with a study conducted by Almeneh K abeta Daba et.al to assess the effectiveness of CMAM(community based management of acute malnutrition program) integrated with nutrition education of knowledge on infant and young child feeding (IYCF) among mothers, care given in Dilla Zuria South Ethiopia which showed that the mothers knowledge before CMAM was low with a mean score 5.39 and post test knowledge score increased to 7.8, which reveals the effectiveness of CMAM.

In the present study, the expressed practice of the mothers was assessed by structure expressed practice questionnaire which revealed that the expressed practice pre test and post test mean score was 49.30 and 59.57 respectively which was consistent with a study conducted by Mastewal Erango Ersado to assess the mothers practice on infant young child feeding showed that mothers practice before intervention was low with 8.27 and post test practice score increased to 9.66 .

In the present study, the sample characteristics socioeconomic status was significantly associated with knowledge which was consistent with a study of Mr. Eptihag Abdelrahman et al. to assess the knowledge of mothers of under five children on Malnutrition and related factors in Kosti and tandalty hospital, White Nile state, Sudan. The findings of study showed that prevalence of under weight decreased significantly as family income increased and higher level of mothers education. The findings of study revealed that there was significant association between knowledge and selected demographic variables.

In present study, total children identified as Stunted were 3(7.1\%) while severe stunted were $1(2.4 \%)$. These findings were inconsistent with the findings of Pascale Vonaesch where it was find that stunted children $51 \%$ (75/148) showed a moderate delay in linear growth for their age group while 49\% (73/148) presented a severe delay.

\section{Conclusion:-}

The CBNP was effective in enhancing knowledge and expressed practices of mothers on prevention of stunted growth. There was significant relationship in the knowledge and expressed practices of mothers on prevention of stunted growth

\section{Recommendations}

1. A study can be replicated on a large sample of rural area of Haryana for wider generalizations of findings.

2. A study be carried out by using other strategies like structured teaching program etc.

3. A comparative study can be conducted to assess knowledge, attitudes and practices of mothers regarding prevention of stunted growth among rural and urban .

4. A study can be conducted on to assess attitude of mothers on prevention of stunted growth.

5. A longitudinal study can be conducted by using "Time series design" to find out the effectiveness of nutritional programme 


\section{Acknowledgement :-}

We are thankful to all the participants of study.

\section{Conflict of Interest}

The authors declare no conflict of interest.

\section{References:-}

1. World Health Organization (WHO) Child Growth Standards: http://www.who.int/childgrowth/en/.

2. National Health Policy, 2017, Ministry of Health and Family Welfare, March 16, 2017, http://mohfw.nic.in/showfile.phplid 4275

3. Gupta MC, Mehrota M, Arora S, Saran M. Relation of childhood nutrition malnutrition to parental education and mothers- nutrition related KAP. Indian Journal of Paediatrics[Internet]. 1991[cited 22 March 2018]; 58(2):269-74. Available from: https://link.springer.com/article/10.1007/BF02751136

4. Bhutta Z, Das JK,Razvi A, Gaffy MF,Walker N,Hortton S,et al. Evidence-based interventions for improvement of maternal and child nutrition: What can be done and at what cost The lancet2013,382,452-77.

5. Awasthi S, Peto R, Pande V, Fletcher R, Read S, Bundy D. Effects of Deworming on Malnourished Preschool Children in India: An Open-Labelled, Cluster-Randomized Trial. PLoS Neglected Tropical Diseases [Internet]. 2008 [cited 22 March 2018]; 2(4):e223.Availablefrom:http://journals.plos.org/plosntds/article?id=10.1371/journal.pntd.0000223

6. Infant and Young Child Feeding - UNICEF data [Internet]. UNICEF data. 2018 [cited 16 March 2018]. Available from: https://data.unicef.org/topic/nutrition/infant-and-young-child-feeding/

7. E.H Ghoneim, Ezzat Amine, An intervention programme for improving the nutritional status of children aged 2-5 years in Alexandria: Eastern Mediterranean health journal[ pubmed];December 10(6): 828-43.

8. Daniel B, Tesfaye N, Mekonin E, Kassa A, Mensur K, et al. (2017) Knowledge and Attitude on Growth Monitoring and its Associated Factors among Mothers/Guardians of Children Less than Two Years in Areka Town, Southern Ethiopia, 2017. J Nutr Disorders Ther 7: 216. doi: 10.4172/2161- 0509.1000216.

9. Edith,M.,\&Priya,1.(2016).Knowledge, attitude and practice (KAP) survey on dietary practices in prevention of malnutrition among mothers of under five children. Manipal Journal of nursing and health sciences,2(2), 19-24.

10. Daba AK, Ersado ME (2015) Impact of Community Based Management of Acute Malnutrition Integrated Nutrition Education on Infant and Young Child Feeding Knowledge and Practice of Mothers or Caregivers in Dilla Zuria Woreda, Southern Ethiopia: A Quasi Experimental Study. Fam Med Med Sci Res 4:190. doi:10.4172/2327-4972.1000190

11. Eptihag Abdelrahman, Kamal Abdelmokaram, Sharafeldeen Idriss and Waleed Aboshora (2017) Assessment of maternal knowledge of under five's children regards malnutrition and related factors in Kosti and Tandalty Hospital, White Nile State, Sudan. BAOJ Nutrition3:033. 